\title{
Using Cloud Computing in Supporting the Management of Travel Agencies
}

\author{
Wael Fouad Demerdash Mohamed
}

Abbasseya Higher Institute for Computers and Commercial Sciences, Egypt

Copyright $@ 2014$ Horizon Research Publishing All rights reserved.

\begin{abstract}
The Cloud has become a new vehicle for delivering resources such as computing and storage to Customers on demand. Rather than being a new technology in itself, the cloud is a new business model wrapped around new technologies such as server virtualization that take advantage of economies of scale and multi-tenancy to reduce the cost of using information technology resources. Rapid development particularly in the field of information technology and the rise of cloud computing of various types widely enables for exchanging data in a more convenient method for the needs of the clients in sectors that operate in the same field, which utilize same work methodologies. This paper aims at shedding light on the importance of using cloud computing in creating a kind of integrity between "Local and International Tourism Agencies" by transforming a given data format to another software on a cloud provider. The concept will be applied on a number of local agencies in Egypt. Using c\# with SQL Database for building a model for interaction and data exchange between the" local and international agencies"
\end{abstract}

Keywords Cloud Computing, Cloud Storage, Virtulaization, Cluster, Hypervisor

\section{Introduction}

'Cloud computing is a model for enabling ubiquitous, convenient, on-demand network access to a shared pool of configurable computing resources (e.g., networks, servers, storage, applications, and services) that can be rapidly provisioned and released with minimal management effort or service provider interaction", "1NIST]

During the past few years, there has been an evident shift in the world of computers and internet functions from the ordinary users' computers to remote servers that form a

\footnotetext{
${ }^{1}$ The National Institute of Standards and Technology (NIST), known between 1901 and 1988 as the National Bureau of Standards (NBS), is a measurement standards laboratory, also known as a National Metrological Institute (NMI), which is a non-regulatory agency of the United States Department of Commerce.
}

cloud of data and data processing. The idea of cloud computing has transferred the traditional concept of data creation and transmission into gates that enable viewing and modifying data that are held under the control of cloud service providers such as Amazon, Windows Azure, IBM, $[1],[2],[3]$

An example of cloud is Google Drive service., It allows users to create and edit documents online while collaborating with other users live. All files created with the apps are by default saved to Google Drive.

Data storage of files was introduced on January 12, 2010, with 1 GB of free space. On April 24, 2012, Google launched Google Drive which supplants Google Docs [4]

\subsection{Modules of Clouds}

Software as a Service: The clients use the cloud network to run a specific computer application, but do not control the infrastructure that runs the application.

A popular example is Google Docs, in which users can build spreadsheets or create documents using Google's software and remote storage space. Face book operates similarly, allowing customers to post photos, messages, through a website; it allows a user to use the provider's application hosted by the cloud vendor on pay-per-use basis. [5]

Platform as a Service: In these module clients uses the cloud network to create and deliver new electronic applications but does not control the cloud's infrastructure.

One famous example is Microsoft Azure, which allows users to build and modify applications and then distribute them to customers. The city of Miami used Azure to build a map tracking information about ${ }^{2}$ potholes, missed garbage collection, and illegal trash dumping [6].

Infrastructure as a Service: using this module provide the customers to buy new hardware from a infrastructure service provider.

For example, if a clothing store needs additional computing power for its website during the holiday shopping

\footnotetext{
${ }^{2}$ Failure in asphalt pavement caused by the presence of water underlying soil structure and the presence of traffic passing over the affected over.
} 
season, it could buy cloud space from companies like Rackspace that provide direct access to cloud infrastructure without buying new hardware.[7] Similarly, NASA also rented computer power from Amazon to process information received from the Mars rovers.

\section{Foundation of Cloud Computing}

\subsection{Virtualizations}

Virtualization is a critical piece of the cloud without it, you cannot realistically provide rapid, flexbility, self-service delivery of services or a collection of consolidated shared resources By isolating services and machines from hardware [8]

The main aim of virtualization technologies is to hide the physical characteristics of computing resources from the way in which other systems, applications, or end users interact with those resources,

It possible for an IT Department to build a library of reusable resources that can be available to the business for building a private cloud [14].

\subsubsection{A virtualization layer (Hypervisor)}

Using VM [9] provides the required infrastructural support and making use of lower-level hardware resources in order to create multiple independent virtual machines that are isolated from each other.

This layer, traditionally Hypervisor, usually sits on top of the hardware and below the operating system.

Hypervisor is a program based on abstractions lying between a real machine and an emulator; in this case a virtual machine is an environment created and managed by a VMM manger.[10]

The Virtual Machine (VM) concept [11] dates back to the 1960s; it was introduced by IBM as a mean to provide concurrent, interactive access to their mainframe computers. A VM was an instance of the physical machine and gave users the illusion of accessing the physical machine directly.

VMM is in charge of managing one or more VMs, and each VM in turn provides facilities to an OS or application as if it is run in a normal environment, directly on the hardware.

Examples include VMware [12], Xen [13], [14], [15], Intel[16]

\subsection{Clustering}

Clustering allows multiple systems to function as one large system by using software and networking technologies such as shared file systems, high-speed interconnects (for servers), and similar technology. This, in turn, helps users to scale out their applications easily by adding more systems to an existing cluster to overcome the limits of physical resources (CPU and memory) in a single server. It also makes the applications highly available by allowing them to run on multiple servers in an active-active fashion to avoid single points of failure $[17,18]$

\subsubsection{Architecture of System Virtualization}

A generic architecture of system virtualization, implemented in these systems, is given in Fig. 1.

The access to CPU resource is native, to all VMs sharing the CPU, for all instructions except the privileged instructions. The privileged instructions are virtualized, i.e.,

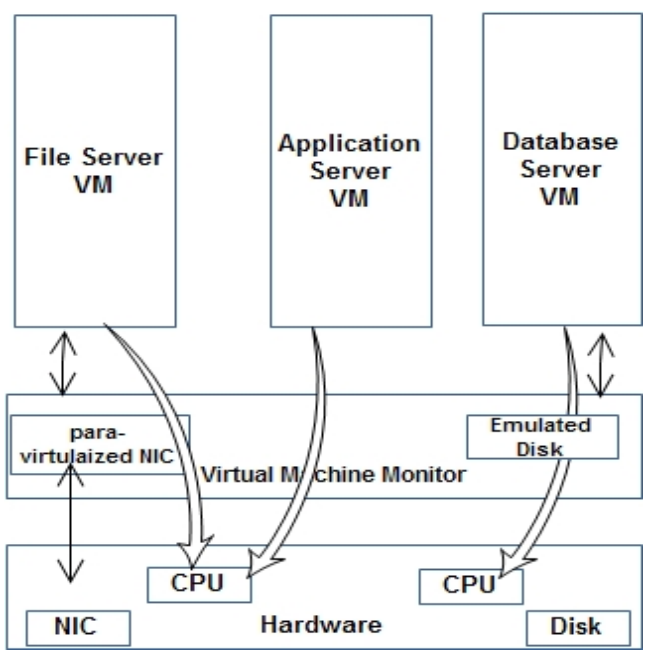

Figure 1. Generic System Virtualization architecture

In Figure 1 Instructions are executed from within the VM, they are trapped, and control is passed to the VMM. All I/O instructions fall under the category of privileged instructions. Thus, I/O devices like the Network Interface Card (NIC) and the DISK are treated differently, when virtualized. There are two different, popularly adopted, methods used for vitalizing I/O devices, namely, para-virtualization and emulation [19].

Both these modes provide data protection and integrity to independent VMs but suffer from loss of performance and usable device bandwidth. [20].

\subsection{Multi-core Processor}

Processors were originally developed with only one core. Multi-core processors were developed in the early 2000s by Intel, AMD and others.

Multi-core processors are widely used across many application domains including general-purpose, embedded, network, digital signal processing (DSP), and graphics. a single computing component with two or more independent actual central processing units (called "cores"), the multiple cores can run multiple instructions at the same time, increasing overall speed for programs amenable to parallel computing. [21],[22]

The improvement in performance gained by the use of a multi-core processor depends very much on the software algorithms used and their implementation. In particular, possible gains are limited by the fraction of the software that can be run in parallel. 


\subsection{Storage}

The capacity of hard drives has grown exponentially over time. With early personal computers, a drive with a 20 megabyte capacity was considered large. In the latter half of the 1990s, hard drives with capacities of 1 gigabyte and [22] greater became available. As of 2006, the "smallest" desktop hard disk still in production has a capacity of 40 gigabytes, while the largest-capacity internal drives are a $3 / 4$ terabyte (750 gigabytes), with external drives at or exceeding one terabyte by using multiple internal disks. These new internal drives increased their storage capacities with Perpendicular recording.

\subsection{Communication}

Bandwidth describes the maximum data transfer rate of a network or Internet connection. It measures how much data can be sent over a specific connection in a given amount of time. For example, a gigabit Ethernet connection has a bandwidth of 1,000 Mbps, (125 megabytes per second). An Internet connection via cable modem may provide $25 \mathrm{Mbps}$ of bandwidth.

\subsection{Type of Cloud}

- Private cloud: a cloud that is used exclusively by one organization. The cloud may be operated by the Organization itself or a third party. The St Andrews Cloud Computing Co-laboratory and Concur Technologies [24] are example organizations that have private clouds.
- Public cloud: a cloud that can be used (for a fee) by the general public. Public clouds require significant investment and are usually owned by large corporations such as Microsoft, Google or Amazon.

- Community cloud: a cloud that is shared by several organizations and is usually setup for their specific requirements. The Open Cirrus cloud test bed could be regarded as a community cloud that aims to support research in cloud computing [25].

- Hybrid cloud: a cloud that is setup using a mixture of the above three deployment models. Each cloud in a hybrid cloud could be independently managed but applications and data would be allowed to move across the hybrid cloud. Hybrid clouds allow cloud bursting to take place, which is where a private cloud can burst-out to a public cloud when it requires more resources.

\section{Research Experiment on "Building a Private Cloud and Using an Integrated Data Mapping System"}

\subsection{Problem Identification}

It is well-acquainted with in travel agencies of medium and big tourism operation that they deal with the foreign tour operators by what is called "Agency File", it encompasses all that is related to the market contracted upon.

One tourism market may have more than one file for instance regarding the French market there is a file for tour operator located in a city and another file in another city, in addition regarding the German market there is a file for tour operator located in one city and another.

\section{Enterprise Tourism company}

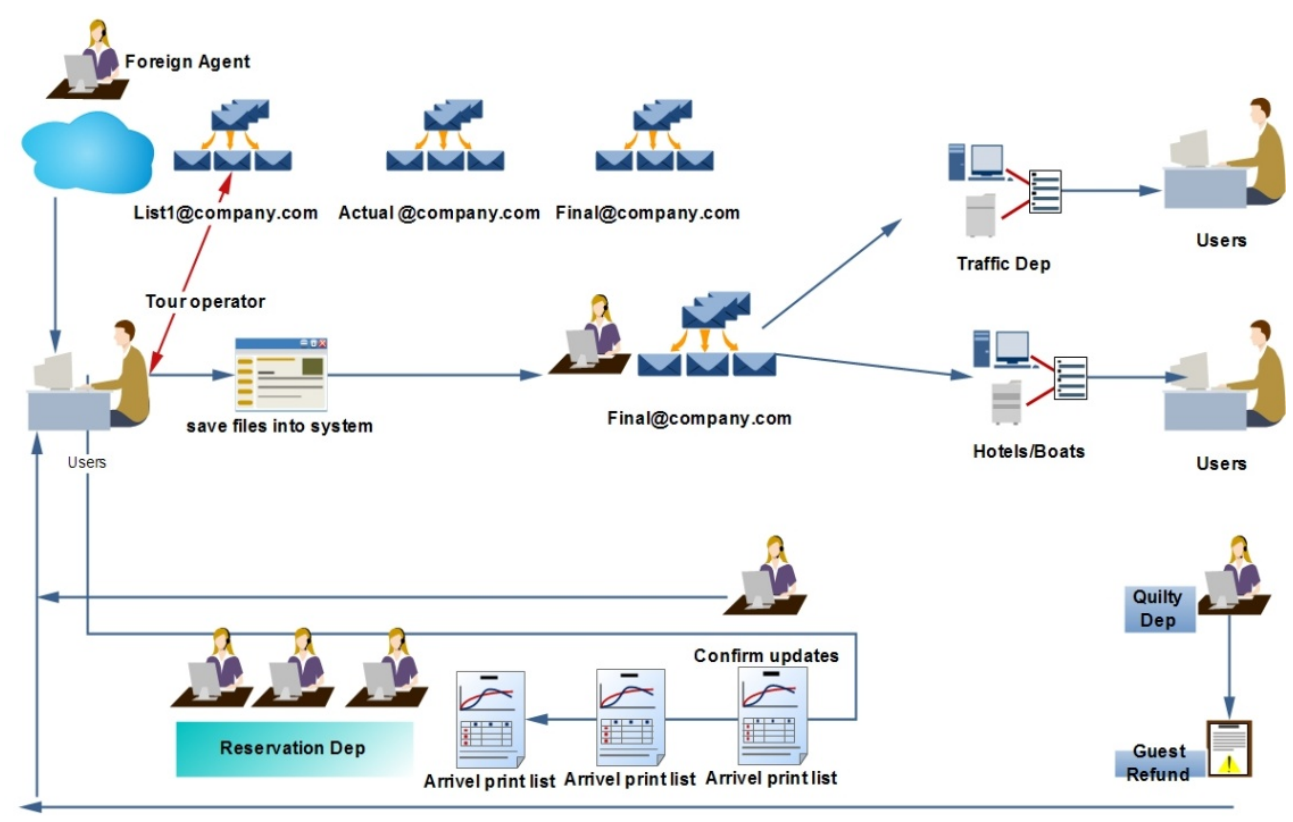

Figure 2. Work Operation company $\mathrm{x}$ 
Accordingly, one local company has the chance of dealing with numerous foreign tour operators, hence arises a difficulty in executing the work requirements for each file individually which necessitates time, effort and numbers of staff; the more the number of foreign files are, the more numbers of executives and computer sets used ,this in turn entails more time and financial load.

In figure 2 which illustrate the work Operation as hereinabove explained.

It is shows that company (x) deal with a number of agents, each agent has its own program; reservation, type of hotel, children policy... etc.

This program is based on the period of stay and excursions. Which enter the tourism programs into the system.

Suppose that there is a given tour operator that sends about 3.000 guests per week, if this number is handled by 5 persons; they handle these 3.000 including accommodation arrangements, type of program, excursions...etc, starting with the initial reservation, then the additions and deletions sent as updates by the foreign tour operators until the final reservations are actually materialized i.e. there are 9000 operations involved. In addition, if the number of names that can be entered into the system per day is one thousand, the staff needs 9 days per week to do this work, which is actually impossible, therefore cloud computing will be utilized for saving and storage, along with a transformation program that handles the process ongoing between the foreign tour operator and the local tour operator in order to solve the aforementioned problem.

\subsubsection{Work Flow}

The process starts by receiving the rooming list from the foreign operator and this is the first time the reservation is conducted. This rooming list contains data such as the full names of the clients, arrival and departure dates, exact itinerary, flight details ...etc.

1) Printing the rooming list.

2) Entering the rooming list to the system.

3) Sending e-mails to hotels, floating hotels, ticketing departments in order to finalize the reservation process.

4) Receiving the respective confirmation from all the departments.

5) If the foreign operator sends any amendment to the above reservation then here arises the problem since the foreign operator will send all the rooming lists again with a any change.

6) These rooming lists will be printed once again by the local operator. A comparison is held by eyesight between the printed rooming lists and the newly received rooming lists with the changes in data regarding the flight lists, hotel change, a guest's name is cancelled or added.

7) After reviewing the new rooming list, the changes are re-entered into the already existing system details.

The cause of the problem here is the fact that this cannot be done in different way, since the foreign operator has a system that sends data as bulk without division or specifications.

\subsubsection{Building Integrated System Application}

The guest should provide us two files one is called structure file and other the data itself Fig 3 contains data send from the foreign tourism agency reservation file.

The tourism agency should send us a structure files

This is a guide to help us where we can start and stop saving the data contain an example for the method would be used for making the integration between the local and foreign agency.

The data format sent by the foreign tour operator, showing the approach for saving the data into the system of local tour Operator.

This table is significant since it is considered a map on which the process of data saving is based.

By using data sent file we can make the date transformation process successfully.

After the clients send the files where in the form on e-mail or through thy portal cloud,

In table 1 illustrates the data format sent by the foreign tour operator, showing the approach for saving the data into the system of local tour Operator.

Table 1. Structure Data File

\begin{tabular}{|c|c|c|c|}
\hline From & To & Colum Name & Agent \\
\hline 1 & 1 & code treatment & 0001 \\
\hline 2 & 3 & Original program code & 0001 \\
\hline 4 & 5 & Document Type & 0001 \\
\hline 6 & 8 & Destination code & 0001 \\
\hline 9 & 9 & Region code & 0001 \\
\hline 10 & 10 & City code & 0001 \\
\hline 11 & 12 & Production Unit Code & 0001 \\
\hline 13 & 14 & Statistics code & 0001 \\
\hline 15 & 17 & Location entry & 0001 \\
\hline 16 & 27 & Input Screen & 0001 \\
\hline 28 & 57 & reference input & 0001 \\
\hline 58 & 67 & profile entry & 0001 \\
\hline 68 & 74 & File Number & 0001 \\
\hline 75 & 82 & Date input file & 0001 \\
\hline 83 & 89 & Original file number & 0001 \\
\hline 90 & 96 & previous piece & 0001 \\
\hline 97 & 103 & Next piece & 0001 \\
\hline 104 & 106 & retrocession & 0001 \\
\hline 106 & 116 & Screen billing & 0001 \\
\hline
\end{tabular}

This table is significant since it is considered a map on which the process of data saving is based.

By using table 1 along with Fig 3 Data sent file we can make the date transformation process successfully. 


\begin{tabular}{|c|c|}
\hline $\begin{array}{l}234476802030630 \mathrm{MMDICARO} \\
000000000000000000000000000000\end{array}$ & 00000000FR2DO TWIN \\
\hline $\begin{array}{rc}234476802130630 M M D I C A R O & \text { CLARISSE } \\
000000000000000000000000000000\end{array}$ & $00000000 \mathrm{FR}$ \\
\hline $\begin{array}{r}234476802230630 \mathrm{MMTAVEL} \\
000000000000000000000000000000\end{array}$ & $00000000 \mathrm{FR} 2 \mathrm{DO}$ \\
\hline $\begin{array}{r}234476802330630 \mathrm{MM} \text { MERNANDEZ } \\
000000000000000000000000000000\end{array}$ & $00000000 \mathrm{FR}$ \\
\hline $\begin{array}{l}234476802430630 \text { MRGAGGIO } \\
000000000000000000000000000000\end{array}$ & $00000000 \mathrm{FR} 2 \mathrm{DO}$ \\
\hline $\begin{aligned} & 234476802530630 M R F A R I N E Y \text { THOMAS } \\
& 000000000000000000000000000000\end{aligned}$ & $00000000 \mathrm{FR}$ \\
\hline $\begin{array}{l}234476802630630 \mathrm{MRAUBERT} \\
000000000000000000000000000000\end{array}$ & $00000000 \mathrm{FR} 2 \mathrm{DO}$ \\
\hline $\begin{array}{r}234476802730630 \mathrm{MMBOEHRINGER} \\
000000000000000000000000000000\end{array}$ & $00000000 \mathrm{FR}$ \\
\hline $\begin{array}{r}234476802830630 \text { MRHESSE } \\
000000000000000000000000000000\end{array}$ & $00000000 \mathrm{FR} 2 \mathrm{DO}$ \\
\hline $\begin{array}{rc}234476802930630 \mathrm{MRTAVEL} & \text { OLIVIER } \\
000000000000000000000000000000\end{array}$ & $00000000 \mathrm{FR}$ \\
\hline $\begin{array}{l}234476803030630 \text { MRDARFEUTLLE } \\
\qquad 00000000000000000000000000000000\end{array}$ & 00000000FR.2DO TWRN \\
\hline $\begin{array}{l}234476803130630 \text { MRDARFEUILLE } \\
\qquad 0000000000000000000000000000000\end{array}$ & $00000000 \mathrm{FR}$ \\
\hline $\begin{array}{l}234476803230630 \mathrm{M} M \text { MULETTA } \\
\qquad 00000000000000000000000000000000\end{array}$ & 00000000FR.2DO \\
\hline
\end{tabular}

Figure 3. Reservation text file

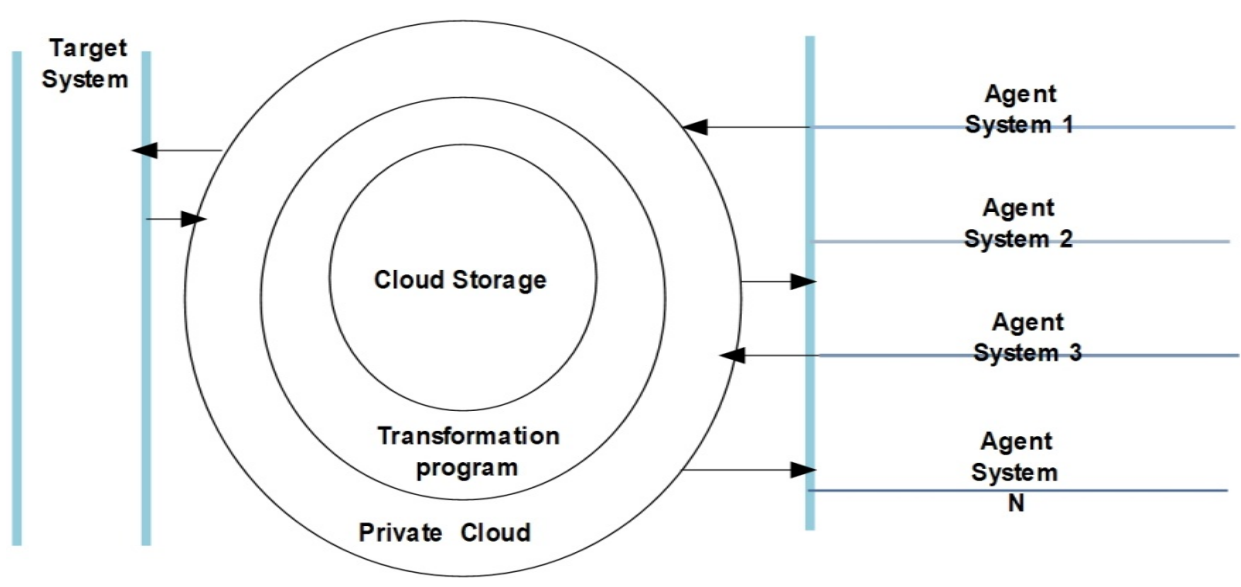

Figure 4. Three layer architecture

\section{For Example}

When we look at table 1 we can see from and to which mean that 68 to 74 means the system has to go to data file in fig 3 starting the space 68 ending the space 74 take the data and put it into the local database system in a reservation table into "file number" we shall apply this on all the elements of the above table, then the following table until we finished all table sent it by the foreign agency.

\section{Operation Code Methodology}

Step 1

Read the "Guest" data file which is come from the foreign agency Fig 3 Illustrated the data which is come into txt file format, each line contains the data belong to specific "Client" so, After we make the first check in order to make sure if the file is corrupted or has anything wrong, we need to put each line in database Colum and record and that leads us to the second step. 


\section{Step 2}

Put the data into record on of them called "Agent Data" and the other called "Agent Id"

Step 3

This step is concerned by using both files Guest database file and structure database file for distributing and match the every single record into the host database file

After terminating the final stage step 3 the model retrieves the files of the clients and initiates the road map of each agent.

This grants the model ability and the flexibility necessary for classifying and organizing the data respective to each agent.

In figure 4 illustrates the Logical Design architecture

The right side shows the multiple agents the local company has to deal with; each agency has its own System regardless of the number of agents involved as long as they all the necessary data is provided.

\section{The Model System is Divided into Three Layer or Stages}

- The private cloud for introduces the all necessary infrastructure to maintain the scalability and availability of the foreign agents.

- Transformation model which is reasonable for maintaining and mapping the data between the foreign agents systems and the local company system.

- The third layer is for providing the travel agencies the storage they are in needed.

- $\quad$ Finally the target system which is for the local agency system that includes the system after the integrated has been successful made.

\subsubsection{Observations after applying the model on one Agent Company}

A relation of direct proportion among three factors: applying the model, time consumption and human factor

Example:

Once an agent sends its files (rooming list, Ticketing, excursion, transportation ...etc), which need 5 dedicated persons working for three full wording day.

But after the model is applied, one employee only is needed and for the first time only to check that all is operating well.

The actual time taken depends on the network bandwidth rate.

The more the number of agents involved is, the better the attained results are

\subsubsection{Building a Private Cloud}

Using Hypervisor for creating your private cloud, it is possible for administrators to combine several VMs on a single server that runs the Hyper-V role. The number of VMs that can be run on a single server varies according to the resources required and the hardware of the physical server that hosts the VMs.

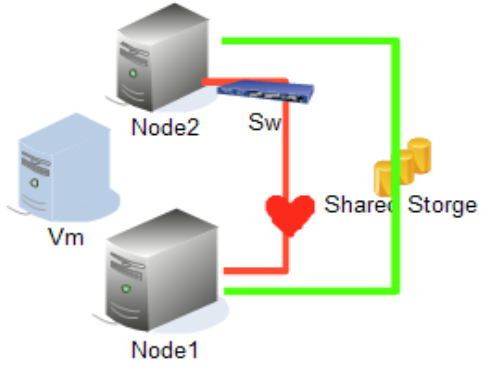

Figure 5. Private Cloud

In the figure 5 there are two computers that are also known as Nodes. The nodes, combined, make up the cluster. Each of the Nodes have at least two network adapters. One of the network Adapters are used to connect each node to the network. The Second network adapter is used to connect the nodes to each Other via a private network, also called a heartbeat network

The private network is used by the cluster service so the nodes Can talk to each other and verify that each other are up and Running. The process of verification uses two processes called the looks alive resource check and the is alive resource check If the resources in the cluster fail to respond to the resource checks after the appropriate amount of time, the passive node will assume that the active node has failed and will start up the service or application that is running on the cluster in the virtual server. Some nodes will also have a third network.

Adapter dedicated to the iSCSI network to access the iSCSI San. Finally by choosing the type of hardware and Virtualizing those using hypervisor and clustering for high availability companied all of this for building the privet cloud.

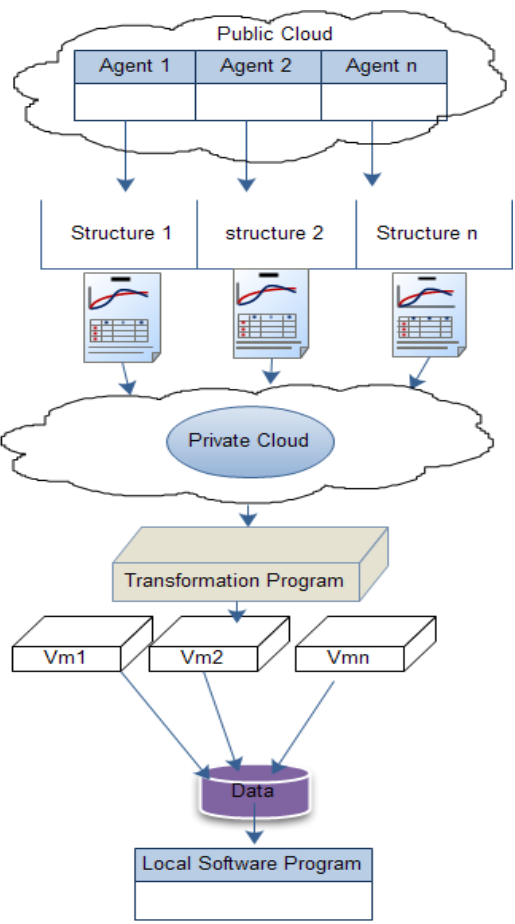

Figure 6. Cloud computing and road map

3.1.5. Design Solution Architecture 
In figure 6 shows the transformation program as a service, deemed as a middle ware which takes the structure files of every agent as a road map on the basis of this structure, thus making the transformation data into the local database agency system.

The VMM virtual machine manger in the private cloud takes care of this issue which VM should be responsible for the data of each agent.

\section{Conclusions}

The overall aim of this paper is to identify how we could use cloud computing for business entities to manage their core business that depends on retrieving flow of information from different business systems characterized with non-similar data format

To fulfill this aim, this paper describes a model which provides a kind of data transformation with widely agreed upon business characteristics information to allow the translation from different systems to the target system, by using cloud storage as a service as first phase, transformation engine as a second phase and private cloud as a third phase. The aforementioned unified integration is hence created. As a result, the top management of any entity utilizing this model will be able to manage its business in a totally different view witnessing many privileges such as cutting the expenses, utilizing the workforce in different way.

It is worth-mentioning that this integration may be applied to any relevant business domain, but in this research the field of tourism has been taken as an example.

\section{REFERENCES}

[1] D. R. Augustyn and L. Warchal, "Volume 79: Cloud Ser-vice Solving N-Body Problem Based on Windows Azure Platform," In: Communications in Computer and Infor-mation Science, Springer, Berlin, Heidelberg, 2010, pp. 84-95.

[2] Amazon, "Amazon Elastic Compute Cloud (Amazon) (EC2),"Journal of Parallel and Distributed Computing, Vol. 68, No. 4, 2008, pp. 399-562.

[3] "What Is Cloud Computing," Journal of Parallel and Distributed Computing, Vol. 68, No. 11, 2008, pp. 1403-1516.

[4] ERDOGMUS, H. 2009. Cloud Computing: Does Nirvana Hide behind the Nebula? Software, IEEE 26, 2, 4-6.

[5] http://microsoft.com/casestudies/Case_Study_Detail.aspx?C aseStudyID $=4000006568$

[6] Rackspace, Cloud Servers, accessed June 20, 2010, available http://www.rackspacecloud.com/cloud_hosting_products/ser vers

[7] M. C. Jaeger, G. Muhl and S. Golze, "QoS-Aware Com-position of Web Services: A Look at Selection Algo-rithms," Proceedings of the IEEE International
Confer-ence on Web Services (ICWS), Orlando, 11-15 July 2005, 1-2.

[8] Vmware (2005) Vmware ESX Server 2-architecture and performance implications (2005). Available online: http://www.vmware.com/pdf/esx2_performance_implication s.pdf. Cited 30 April 2010

[9] Lakshmi, J., Nandy, S.K.: Quality of service for I/O workloads in multicore virtualized servers. In: Cafaro, M., Aloisio, G. (eds.) Grids, Clouds and Virtualization. Springer, Berlin (2010)

[10] Krishnan, S.: Programming Windows Azure. O’Reilly Media, New York (2010)

[11] Nanda, S., Cker Chiueh, T.: A survey of virtualization technologies. Tech. rep., State Univer-sity of New York at Stony Brook (2005)

[12] Scott,l.and Nick,M.(2014) Mastering Vmware vsphere5.5.Canda:Chris.W Sybex ISBN:978-1-118-66114-7.

[13] P. Barham, B. Dragovic, K. Fraser, S. Hand, T. Harris, A. Ho, R. Neugebauer, I. Pratt, and A. Warfield. Xen and the art of virtualization. In Proc. of 19th ACM SOSP, New York, NY, Oct 2003

[14] J. Carolan and S. Gaede, "Introduction to Cloud Comput-ing Architecture, Sun Microsystems Inc. White Paper," Sun Microsystems Inc., Santa Clara, 2009.

[15] Wikipedia, "Wikipedia, Free Encyclopedia. 3rd Interna-tional Conference on Grid and Pervasive

Computing-gpc-Workshops," 2008. www.wikipedia.com Intel Virtualization Technology for Directed-I/O (2006).

[16] Available online: www.intel.com/ technology/itj/2006/v10i3/2-io/7-conclusion.htm. Cited 30 April 2010

[17] Chase, J.S., Irwin, D.E., Grit, L.E., Moore, J.D., Sprenkle, S.E.: Dynamic virtual clusters in a grid site manager. In: Proceedings of the 12th International Symposium on High Performance Distributed Computing (HPDC 2003) (2003)

[18] Emeneker, W., Jackson, D., Butikofer, J., Stanzione, D.: Dynamic virtual clustering with Xen and Moab. In: Proceedings of the Frontiers of High Performance Computing and Networking, ISPA 2006 Workshops. Lecture Notes in Computer Science, vol. 4331, pp. 440-451. Springer, Berlin (2006)

[19] Rixner, S.: Breaking the performance barrier: shared I/O in virtualization platforms has come a long way but performance concerns remain. ACM Queue 6(1), 36 (2008)

[20] SunMicrosystems: CrossBow Network Virtualization and Resource Control (2008). Available online: http://www.opensolaris.org/os/community/networking/cross bow_sunlabs_ ext.pdf. Cited 30 April 2010

[21] URL http://en.wikipedia.org/wiki/Multi-core_processor

[22] Lakshmi, J., Nandy, S.K.: I/O device virtualization in multi-core era, a QoS perspective. In: Workshop on Grids, Clouds and Virtualization, Conference on Grids and Pervasive Computing, pp. 128-135 (2009)

[23] URLhttp://en.wikibooks.org/wiki/The_Computer_Revolutio $\mathrm{n} /$ Hardware/Storage

[24] LEMOS, R. 2009. Inside One Firm's Private Cloud Journey. 
Retrieved December 1, 2009, from http://www.cio.com/article/506114/Inside_One_Firm_s_Pri vate_Cloud_Journey
[25] Open CirrusTM: the HP/Intel/Yahoo! Open Cloud Computing Research Testbed. Retrieved December 1, 2009, from https://opencirrus.org/ 